\title{
Compositional and holistic quantum computational semantics
}

\author{
MARIA LUISA DALLA CHIARA ${ }^{1, *}$, ROBERTO GIUNTINI $^{2}$ \\ and ROBERTO LEPORINI ${ }^{3}$ \\ ${ }^{1}$ Dipartimento di Filosofia, Università di Firenze, via Bolognese 52, I-50139, Firenze, Italy \\ (*Author for correspondence, e-mail: dallachiara@unifi.it); ${ }^{2}$ Dipartimento di Scienze \\ Pedagogiche e Filosofiche, Università di Cagliari, via Is Mirrionis 1, I-09123, Cagliari, \\ Italy; ${ }^{3}$ Dipartimento di Matematica, Statistica, Informatica e Applicazioni, Università di \\ Bergamo, via dei Caniana 2, I-24127, Bergamo, Italy
}

\begin{abstract}
In quantum computational logic meanings of sentences are identified with quantum information quantities: systems of qubits or, more generally, mixtures of systems of qubits. We consider two kinds of quantum computational semantics: (1) a compositional semantics, where the meaning of a compound sentence is determined by the meanings of its parts; (2) a holistic semantics, which makes essential use of the characteristic "holistic" features of the quantum-theoretic formalism. We prove that the compositional and the holistic semantics characterize the same logic.
\end{abstract}

Key words: quantum computation, quantum logic

\section{Introduction}

The main difference between orthodox quantum logic [first proposed by Birkhoff and von Neumann (1936)] and quantum computational logic (Dalla Chiara et al., 2003) concerns a basic semantic question: how to represent the meanings of the sentences of a given language? The answer given by Birkhoff and von Neumann was the following: the meanings of the elementary experimental sentences of quantum theory have to be regarded as determined by appropriate sets of states of quantum objects (mathematically represented by closed subspaces of a Hilbert space). The answer given in the framework of quantum computational logics is quite different. The meaning of a sentence is identified with a quantum information quantity: a system of qubits or, more generally, a mixture of systems of qubits. 
We will consider two kinds of quantum computational semantics:

- A compositional semantics, where (like in classical logic) the meaning of a compound sentence is determined by the meanings of its parts.

- A holistic semantics, which makes essential use of the characteristic "holistic" features of the quantum-theoretic formalism. Hence, in this framework, the meaning of a compound sentence generally determines the meanings of its parts, but not the other way around.

We will prove that the compositional and the holistic semantics characterize the same logic.

\section{Qubits, quregisters and qumixes}

Let us first recall some basic definitions of quantum computation. Consider the two-dimensional Hilbert space $\mathbb{C}^{2}$ (where any vector $|\psi\rangle$ is represented by a pair of complex numbers). Let $\mathcal{B}^{(1)}=\{|0\rangle,|1\rangle\}$ be the canonical orthonormal basis for $\mathbb{C}^{2}$, where $|0\rangle=(1,0)$ and $|1\rangle=(0,1)$.

Definition 2.1 (Qubit). A qubit is a unit vector $|\psi\rangle$ of the Hilbert space $\mathbb{C}^{2}$.

From an intuitive point of view, a qubit can be regarded as a quantum variant of the classical notion of bit: a kind of "quantum perhaps". In this framework, the two basis-elements $|0\rangle$ and $|1\rangle$ represent the two classical bits 0 and 1, respectively. From a physical point of view, a qubit represents a state of a single particle, carrying an atomic piece of quantum information. In order to carry the information stocked by $n$ qubits, we need of course a compound system, consisting of $n$ particles.

Definition 2.2 (Quregister). An $n$-qubit system (also called n-quregister) is a unit vector in the $n$-fold tensor product Hilbert space $\otimes^{n} \mathbb{C}^{2}:=\underbrace{\mathbb{C}^{2} \otimes \cdots \otimes \mathbb{C}^{2}}_{n \text {-times }}$ (where $\otimes 1 \mathbb{C}^{2}:=\mathbb{C}^{2}$ ).

We will use $x, y, \ldots$ as variables ranging over the set $\{0,1\}$. At the same time, $|x\rangle,|y\rangle, \ldots$ will range over the basis $\mathcal{B}^{(1)}$. Any factorized unit vector $\left|x_{1}\right\rangle \otimes \cdots \otimes\left|x_{n}\right\rangle$ of the space $\otimes^{n} \mathbb{C}^{2}$ will be called a classical register. Instead of $\left|x_{1}\right\rangle \otimes \cdots \otimes\left|x_{n}\right\rangle$ we will simply write $\left|x_{1}, \ldots, x_{n}\right\rangle$. 
The set $\mathcal{B}^{(n)}$ of all classical registers is an orthonormal basis for the space $\otimes^{n} \mathbb{C}^{2}$.

Quregisters are pure states: maximal pieces of information about the particles under consideration. Both in quantum theory and in quantum information, one cannot help referring also to mixed states (or mixtures), which represent pieces of information that are not maximal and might be enriched. In the framework of quantum computation, mixed states (mathematically represented by density operators of an appropriate Hilbert space) are also called qumixes.

Definition 2.3 (Qumix). A qumix is a density operator of $\otimes^{n} \mathbb{C}^{2}$ (where $n \geq 1)$.

Needless to say, quregisters correspond to particular qumixes that are pure states (i.e. projections onto one-dimensional closed subspaces of a given $\left.\otimes^{n} \mathbb{C}^{n}\right)$. We will indicate by $\mathfrak{D}\left(\otimes^{n} \mathbb{C}^{2}\right)$ the set of all density operators of $\otimes^{n} \mathbb{C}^{2}$. Hence the set $\mathfrak{D}=\bigcup_{n=1}^{\infty} \mathfrak{D}\left(\otimes^{n} \mathbb{C}^{2}\right)$ will represent the set of all possible qumixes.

A classical register $\left|x_{1}, \ldots, x_{n}\right\rangle$ is called true, when $x_{n}=1$; false, otherwise. The idea is that any classical register corresponds to a classical truth-value that is determined by its last element. Hence, in particular, the bit $|1\rangle$ corresponds to the truth-value Truth, while the bit $|0\rangle$ corresponds to the truth-value Falsity.

On this basis, we can identify, in any space $\otimes^{n} \mathbb{C}^{2}$, two special projection-operators $\left(P_{1}{ }^{(n)}\right.$ and $\left.P_{0}{ }^{(n)}\right)$ that represent, in this framework, the Truth-property and the Falsity-property, respectively. The projection $P_{1}{ }^{(n)}$ is determined by the closed subspace spanned by the set of all true registers, while $P_{0}{ }^{(n)}$ is determined by the closed subspace spanned by the set of all false registers. As is well known, in quantum theory, projections have the role of mathematical representatives of possible physical properties of the quantum objects under investigation. Hence, it turns out that Truth and Falsity behave here as special cases of physical properties.

As a consequence, one can naturally apply the Born rule that determines the probability-value that a quantum system in a given state satisfies a given property. Consider any qumix $\rho$, which represents a possible state of a quantum system in the space $\otimes^{n} \mathbb{C}^{2}$. By applying the Born rule, we obtain that the probability-value that a physical system in state $\rho$ satisfies the Truth-property $P_{1}^{(n)}$ is the number $\operatorname{tr}\left(P_{1}^{(n)} \rho\right)$ (where $\operatorname{tr}$ is the trace functional). This suggests the 
following natural definition of the notion of probability of a given qumix.

Definition 2.4 (Probability of a qumix). For any qumix $\rho \in \mathfrak{D}\left(\otimes^{n} \mathbb{C}^{2}\right)$ :

$$
\mathrm{p}(\rho)=\operatorname{tr}\left(P_{1}^{(n)} \rho\right)
$$

From an intuitive point of view, $\mathrm{p}(\rho)$ represents the probability that the information stocked by the qumix $\rho$ is true. In the particular case where $\rho$ corresponds to the qubit

$$
|\psi\rangle=c_{0}|0\rangle+c_{1}|1\rangle
$$

we obtain that $\mathrm{p}(\rho)=\left|c_{1}\right|^{2}$.

Given a quregister $|\psi\rangle$, we will also write $\mathrm{p}(|\psi\rangle)$ instead of $\mathrm{p}\left(P_{|\psi\rangle}\right)$, where $P_{|\psi\rangle}$ is the density operator represented by the projection onto the one-dimensional subspace spanned by the vector $|\psi\rangle$.

\section{Quantum gates}

In quantum computation, quantum logical gates (briefly, gates) are unitary operators that transform quregisters into quregisters. Being unitary, gates represent characteristic reversible transformations. The canonical gates (which are studied in the literature) can be naturally generalized to qumixes. Generally, gates correspond to some basic logical operations that admit a reversible behaviour. We will consider here the following gates: the negation, the Petri-Toffoli gate and the square root of the negation. Let us first describe these gates in the framework of quregisters.

Definition 3.1 (The negation). For any $n \geq 1$, the negation on $\otimes^{n} \mathbb{C}^{2}$ is the linear operator $\operatorname{Not}^{(n)}$ such that for every element $\left|x_{1}, \ldots, x_{n}\right\rangle$ of the basis $\mathcal{B}^{(n)}$ :

$$
\operatorname{Not}^{(n)}\left(\left|x_{1}, \ldots, x_{n}\right\rangle\right):=\left|x_{1}, \ldots, x_{n-1}\right\rangle \otimes\left|1-x_{n}\right\rangle
$$

In other words, $\operatorname{Not}^{(n)}$ inverts the value of the last element of any basis-vector of $\otimes^{n} \mathbb{C}^{2}$. 
Definition 3.2 (The Petri-Toffoli gate). For any $n \geq 1$ and any $m \geq 1$ the Petri-Toffoli gate is the linear operator $\mathrm{T}^{(n, m, 1)}$ defined on $\otimes^{n+m+1} \mathbb{C}^{2}$ such that for every element $\left|x_{1}, \ldots, x_{n}\right\rangle \otimes\left|y_{1}, \ldots, y_{m}\right\rangle \otimes|z\rangle$ of the basis $\mathcal{B}^{(n+m+1)}$ :

$$
\begin{aligned}
\mathrm{T}^{(n, m, 1)}\left(\left|x_{1}, \ldots, x_{n}\right\rangle \otimes\left|y_{1}, \ldots, y_{m}\right\rangle \otimes|z\rangle\right):= & \left|x_{1}, \ldots, x_{n}\right\rangle \\
& \otimes\left|y_{1}, \ldots, y_{m}\right\rangle \otimes\left|x_{n} y_{m} \oplus z\right\rangle,
\end{aligned}
$$

where $\oplus$ represents the sum modulo 2 .

One can easily show that both $\operatorname{Not}^{(n)}$ and $\mathrm{T}^{(n, m, 1)}$ are unitary operators.

Consider now the set $\mathfrak{R}$ of all quregisters $|\psi\rangle$ "living" in $\otimes^{n} \mathbb{C}^{2}$, for an $n \geq 1$. The gates Not and $\mathrm{T}$ can be uniformly defined on this set in the expected way:

$$
\begin{array}{r}
\operatorname{Not}(|\psi\rangle):=\operatorname{Not}^{(n)}(|\psi\rangle), \quad \text { if }|\psi\rangle \in \otimes^{n} \mathbb{C}^{2} ; \\
\mathrm{T}(|\psi\rangle \otimes|\varphi\rangle \otimes|\chi\rangle):=\mathrm{T}^{(n, m, 1)}(|\psi\rangle \otimes|\varphi\rangle \otimes|\chi\rangle), \\
\text { if }|\psi\rangle \in \otimes^{n} \mathbb{C}^{2},|\varphi\rangle \in \otimes^{m} \mathbb{C}^{2} \text { and }|\chi\rangle \in \mathbb{C}^{2} .
\end{array}
$$

On this basis, a conjunction And, a disjunction Or, can be defined for any pair of quregisters $|\psi\rangle$ and $|\phi\rangle$ :

$$
\begin{aligned}
& \operatorname{And}(|\psi\rangle,|\varphi\rangle):=\mathrm{T}(|\psi\rangle \otimes|\varphi\rangle \otimes|0\rangle) \\
& \operatorname{Or}(|\psi\rangle,|\varphi\rangle):=\operatorname{Not}(\operatorname{And}(\operatorname{Not}(|\psi\rangle), \operatorname{Not}(|\varphi\rangle))) .
\end{aligned}
$$

Clearly, $|0\rangle$ represents an "ancilla" in the definition of And.

The quantum logical gates we have considered so far are, in a sense, "semiclassical". A quantum logical behaviour only emerges in the case where our gates are applied to superpositions. When restricted to classical registers, such operators turn out to behave as classical (reversible) truth-functions. We will now consider an important example of a genuine quantum gate that transforms classical registers (elements of $\mathcal{B}^{(n)}$ ) into quregisters that are superpositions. This gate is the square root of the negation. 
Definition 3.3 (The square root of the negation). For any $n \geq 1$, the square root of the negation on $\otimes^{n} \mathbb{C}^{2}$ is the linear operator $\sqrt{\operatorname{Not}}(n)$ such that for every element $\left|x_{1}, \ldots, x_{n}\right\rangle$ of the basis $\mathcal{B}^{(n)}$ :

$$
\begin{aligned}
\sqrt{\operatorname{Not}}^{(n)}\left(\left|x_{1}, \ldots, x_{n}\right\rangle\right):= & \left|x_{1}, \ldots, x_{n-1}\right\rangle \otimes \frac{1}{2}\left((1+i)\left|x_{n}\right\rangle\right. \\
& \left.+(1-i)\left|1-x_{n}\right\rangle\right)
\end{aligned}
$$

where $i:=\sqrt{-1}$.

One can easily show that $\sqrt{\mathrm{Not}}^{(n)}$ is a unitary operator. The basic property of $\sqrt{\operatorname{Not}}^{(n)}$ is the following:

$$
\text { for any }|\psi\rangle \in \otimes^{n} \mathbb{C}^{2}, \sqrt{\operatorname{Not}}^{(n)}\left(\sqrt{\operatorname{Not}}^{(n)}(|\psi\rangle)\right)=\operatorname{Not}^{(n)}(|\psi\rangle) \text {. }
$$

In other words, applying twice the square root of the negation means negating.

From a logical point of view, $\sqrt{\operatorname{Not}}^{(n)}$ can be regarded as a "tentative partial negation" (a kind of "half negation") that transforms precise pieces of information into maximally uncertain ones. For, we have:

$$
\mathrm{p}\left(\sqrt{\operatorname{Not}}^{(1)}(|1\rangle)\right)=\frac{1}{2}=\mathrm{p}\left(\sqrt{\operatorname{Not}}^{(1)}(|0\rangle)\right) .
$$

As expected, also $\sqrt{\text { Not }}$ can be uniformly defined on the set $\mathfrak{R}$ of all quregisters.

Interestingly enough, the gate $\sqrt{\text { Not }}$ seems to represent atypically quantum logical operation that does not admit any counterpart either in classical logic or in standard fuzzy logics (see Dalla Chiara et al., 2005).

The gates considered so far can be naturally generalized to qumixes (Gudder, 2003). When our gates will be applied to density operators, we will write: NOT, $\sqrt{\mathrm{NOT}}, \mathbb{T}, \mathrm{AND}, \mathrm{OR}$ (instead of Not, $\sqrt{\mathrm{Not}}$, $\mathrm{T}$, And, Or).

Definition 3.4 (The negation). For any qumix $\rho \in \mathfrak{D}\left(\otimes^{\mathfrak{n}} \mathbb{C}^{2}\right)$,

$$
\operatorname{NOT}^{(n)}(\rho):=\operatorname{Not}^{(n)} \rho \operatorname{Not}^{(n)} .
$$




\section{QUANTUM COMPUTATIONAL SEMANTICS}

Definition 3.5 (The square root of the negation). For any qumix $\rho \in \mathfrak{D}\left(\otimes^{n} \mathbb{C}^{2}\right)$,

$$
\sqrt{\mathrm{NOT}}^{(n)}(\rho):=\sqrt{\operatorname{Not}}^{(n)} \rho \sqrt{\operatorname{Not}}^{(n) *},
$$

where $\sqrt{\operatorname{Not}}^{(n) *}$ is the adjoint of $\sqrt{\operatorname{Not}}^{(n)}$.

It is easy to see that for any $n \in \mathbb{N}^{+}$, both $\operatorname{NOT}^{(n)}(\rho)$ and $\sqrt{\mathrm{NOT}}^{(n)}(\rho)$ are qumixes of $\mathfrak{D}\left(\otimes^{n} \mathbb{C}^{2}\right)$.

Definition 3.6 (The conjunction). Let $\rho \in \mathfrak{D}\left(\otimes^{\mathfrak{n}} \mathbb{C}^{2}\right)$ and $\sigma \in \mathfrak{D}\left(\otimes^{m} \mathbb{C}^{2}\right)$.

$$
\operatorname{AND}^{(n, m, 1)}(\rho, \sigma)=\mathbb{T}^{(n, m, 1)}\left(\rho, \sigma, P_{0}^{(1)}\right):=\mathrm{T}^{(n, m, 1)}\left(\rho \otimes \sigma \otimes P_{0}^{(1)}\right) \mathrm{T}^{(n, m, 1)} .
$$

Like in the quregister-case, the gates NOT, $\sqrt{\mathrm{NOT}}, \mathbb{T}$, AND, OR can be uniformly defined on the set $\mathfrak{D}$ of all qumixes.

An interesting preorder relation can be defined on the set of all qumixes.

Definition 3.7 (Preorder). $\rho \preceq \sigma$ iff the following conditions hold:

(i) $\mathrm{p}(\rho) \leq \mathrm{p}(\sigma)$;

(ii) $\mathrm{p}(\sqrt{\mathrm{NOT}}(\sigma)) \leq \mathrm{p}(\sqrt{\mathrm{NOT}}(\rho))$.

One immediately shows that $\preceq$ is reflexive and transitive, but not antisymmetric. Counterexamples can be easily found in $\mathfrak{D}\left(\mathbb{C}^{2}\right)$.

\section{The compositional quantum computational semantics}

Both the compositional and the holistic semantics are based on the following intuitive idea: any sentence $\alpha$ of the language is interpreted as an appropriate qumix, that generally depends on the logical form of $\alpha$; at the same time, the logical connectives are interpreted as special operations defined in terms of gates. We will consider a minimal (sentential) quantum computational language $\mathcal{L}$ that contains a privileged atomic sentence $\mathbf{f}$ (whose intended interpretation is the truth-value Falsity) and the following primitive connectives: the negation $(\neg)$, the square root of the negation $(\sqrt{\neg}$ ), a ternary conjunction $\bigwedge$ (which corresponds to the Petri-Toffoli gate). For any sentences $\alpha$ and $\beta$, the expression $\Lambda(\alpha, \beta, \mathbf{f})$ is a sentence of $\mathcal{L}$. In this framework, the usual 
conjunction $\alpha \wedge \beta$ is dealt with as metalinguistic abbreviation for the ternary conjunction $\Lambda(\alpha, \beta, \mathbf{f})$.

Let Form $^{\mathcal{L}}$ be the set of all sentences (formulas) of $\mathcal{L}$. We will use the following metavariables: $\mathbf{q}, \mathbf{r} \ldots$ for atomic sentences and $\alpha, \beta, \ldots$ for sentences. The connective disjunction $(\vee)$ is supposed to be defined via de Morgan $(\alpha \vee \beta:=\neg(\neg \alpha \wedge \neg \beta)$ ). This minimal quantum computational language can be extended to richer languages containing other primitive connectives.

We will first introduce the notion of compositional quantum computational model (briefly, compositional QC-model or qumix-model).

Definition 4.1 (Compositional QC-model). A compositional QC-model of $\mathcal{L}$ is a map Qum that associates a qumix to any sentence $\alpha$ of $\mathcal{L}$, satisfying the following conditions:

$$
\operatorname{Qum}(\alpha):=\left\{\begin{array}{cl}
\text { a density operator of } \mathfrak{D}\left(\mathbb{C}^{2}\right) & \text { if } \alpha \text { is an atomic sentence; } \\
P_{0} & \text { if } \alpha=\mathbf{f} ; \\
\operatorname{NOT}(\operatorname{Qum}(\beta)) & \text { if } \alpha=\neg \beta ; \\
\sqrt{\operatorname{NOT}(\operatorname{Qum}(\beta))} & \text { if } \alpha=\sqrt{ } \beta \\
\mathbb{T}(\operatorname{Qum}(\beta), \operatorname{Qum}(\gamma), \operatorname{Qum}(\mathbf{f})) & \text { if } \alpha=\bigwedge(\beta, \gamma, \mathbf{f}) .
\end{array}\right.
$$

The concept of compositional QC-model seems to have a "quasi intensional" feature: the meaning Qum $(\alpha)$ of the sentence $\alpha$ partially reflects the logical form of $\alpha$. In fact, the dimension of the Hilbert space where Qum $(\alpha)$ "lives" depends on the number of occurrences of atomic sentences in $\alpha$.

Definition 4.2 (The atomic complexity of $\alpha$ ).

$$
A t(\alpha)= \begin{cases}1 & \text { if } \alpha \text { is an atomic sentence; } \\ A t(\beta) & \text { if } \alpha=\neg \beta \text { or } \alpha=\sqrt{\neg} \beta \\ A t(\beta)+A t(\gamma)+1 & \text { if } \alpha=\bigwedge(\beta, \gamma, \mathbf{f})\end{cases}
$$

Lemma 4.1 If $\operatorname{At}(\alpha)=n$, then $\operatorname{Qum}(\alpha) \in \mathfrak{D}\left(\otimes^{\mathfrak{n}} \mathbb{C}^{2}\right)$.

We can say that the space $\otimes^{A t(\alpha)} \mathbb{C}^{2}$ represents the semantic space where all possible meanings of $\alpha$ should "live". Accordingly we will also write $\mathcal{H}^{\alpha}$ instead of $\otimes^{A t(\alpha)} \mathbb{C}^{2}$. 
Given a model Qum, any sentence $\alpha$ has a natural probabilityvalue, which can be also regarded as its extensional meaning with respect to Qum.

Definition 4.3 (The probability-value of $\alpha$ in a model Qum).

$$
\operatorname{PQum}_{\mathrm{Qum}}(\alpha):=\mathrm{p}(\mathrm{Qum}(\alpha))
$$

As we already know, qumixes are preordered by the relation $\preceq$. This suggests a natural definition of a logical consequence relation.

Definition 4.4 (Consequence in a model Qum). A sentence $\beta$ is a consequence in a model Qum of a sentence $\alpha\left(\alpha \models_{\text {Qum }} \beta\right)$ iff $\operatorname{Qum}(\alpha) \preceq \operatorname{Qum}(\beta)$.

Definition 4.5 (Logical consequence). A sentence $\beta$ is a logical consequence of a sentence $\alpha(\alpha \models \beta)$ iff for any model Qum, $\alpha \models_{\text {Qum }} \beta$.

We call quantum computational logic (QCL) the logic that is semantically characterized by the logical consequence relation we have just defined. Hence, $\beta$ is a logical consequence of $\alpha$ in the logic QCL $\left(\alpha \models_{\text {QCL }} \beta\right)$ iff $\beta$ is a consequence of $\alpha$ in any model Qum.

So far we have considered models, where the meaning of any sentence is represented by a qumix. A natural question arises: do density operators have an essential role in characterizing the logic QCL? This question has a negative answer. In fact, one can prove that quregisters are sufficient for our logical aims in the case of the minimal quantum computational language $\mathcal{L}$.

Let us first introduce the notion of qubit-model.

Definition 4.6 (Qubit-model). A qubit-model of $\mathcal{L}$ is a function Qub that associates a quregister to any sentence $\alpha$ of $\mathcal{L}$, satisfying the following conditions:

$$
\operatorname{Qub}(\alpha):=\left\{\begin{array}{cl}
\text { a qubit in } \mathbb{C}^{2} & \text { if } \alpha \text { is an atomic sentence; } \\
|0\rangle & \text { if } \alpha=\mathbf{f} ; \\
\operatorname{Not}(\operatorname{Qub}(\beta)) & \text { if } \alpha=\neg \beta ; \\
\sqrt{\operatorname{Not}(\operatorname{Qub}(\beta))} & \text { if } \alpha=\sqrt{\neg} \beta ; \\
\mathrm{T}(\operatorname{Qub}(\beta), \operatorname{Qub}(\gamma), \operatorname{Qub}(\mathbf{f})) & \text { if } \alpha=\bigwedge(\beta, \gamma, \mathbf{f}) .
\end{array}\right.
$$


The notions of consequence and logical consequence are defined like in the case of qumix-models, mutatis mutandis.

One can prove that the qubit-semantics and the qumix-semantics characterize the same logic (see Dalla Chiara et al., 2003).

Quantum computational logic turns out to be a non-standard form of quantum logic. Conjunction and disjunction do not correspond to lattice operations, because they are not generally idempotent $\left(\alpha \models_{\text {QCL }} \alpha \wedge \alpha, \alpha \vee \alpha \models_{\text {QCL }} \alpha\right)$. Unlike Birkhoff and von Neumann's quantum logic, the weak distributivity principle breaks down $\left((\alpha \wedge \beta) \vee(\alpha \wedge \gamma) \models_{\mathbf{Q C L}} \alpha \wedge(\beta \vee \gamma)\right)$. At the same time, the strong distributivity, that is violated in orthodox quantum logic, is here valid $(\alpha \wedge(\beta \vee \gamma) \models$ QCL $(\alpha \wedge \beta) \vee(\alpha \wedge \gamma))$. Both the excluded middle and the non-contradiction principles are violated. As a consequence, one can say that the logic arising from quantum computation represents, in a sense, a new example of fuzzy logic.

The axiomatizability of QCL is an open problem.

\section{Quantum trees}

The meaning and the probability-value of any sentence $\alpha$ can be naturally described (and calculated) by means of a special configuration called quantum tree, that illustrates a kind of reversible transformation of the atomic subformulas of $\alpha$.

The notion of quantum tree can be dealt with either in the framework of the qubit-semantics or in the framework of the qumixsemantics. In the first case quantum trees will be called qubit trees, while in the second case we will speak of qumix trees. Before dealing with quantum trees, we will first introduce the notion of syntactical tree of a sentence $\alpha$ (abbreviated as STree ${ }^{\alpha}$ ). Consider all subformulas of $\alpha$.

Any subformula may be:

- an atomic sentence $\mathbf{q}$ (possibly $\mathbf{f}$ );

- a negated sentence $\neg \beta$;

- a square-root negated sentence $\sqrt{\neg} \beta$;

- a conjunction $\bigwedge(\beta, \gamma, \mathbf{f})$.

The concept of syntactical tree can be illustrated as follows. Every occurrence of a subformula of $\alpha$ gives rise to a node of STree ${ }^{\alpha}$. The tree consists of a finite number of levels and each level is represented by a sequence of subformulas of $\alpha$ : 


$$
\begin{gathered}
\text { Level }_{k}(\alpha) \\
\vdots \\
\text { Level }_{1}(\alpha)
\end{gathered}
$$

The bottom-level (denoted by Level $_{1}(\alpha)$ ) consists of $\alpha$. From each node of the tree at most 3 edges may branch according to the branchingrule (Figure 1). The second level $\left(\operatorname{Level}_{2}(\alpha)\right)$ is the sequence of subformulas of $\alpha$ that is obtained by applying the branching-rule to $\alpha$. The third level $\left(\operatorname{Level}_{3}(\alpha)\right)$ is obtained by applying the branching-rule to each element (node) of $\operatorname{Level}_{2}(\alpha)$, and so on. Finally, one obtains a level represented by the sequence of all atomic occurrences of $\alpha$. This represents the top-level of STree ${ }^{\alpha}$. The height of Stree ${ }^{\alpha}$ (denoted by Height $(\alpha)$ ) is then defined as the number of levels of STree ${ }^{\alpha}$.

As an example, consider the following sentence:

$\alpha=\mathbf{q} \wedge \neg \mathbf{q}=\bigwedge(\mathbf{q}, \neg \mathbf{q}, \mathbf{f})$. The syntactical tree of $\alpha$ is the following:

$$
\begin{aligned}
& \text { Level }_{3}(\alpha)=(\mathbf{q}, \mathbf{q}, \mathbf{f}) ; \\
& \text { Level }_{2}(\alpha)=(\mathbf{q}, \neg \mathbf{q}, \mathbf{f}) ; \\
& \text { Level }_{1}(\alpha)=\bigwedge(\mathbf{q}, \neg \mathbf{q}, \mathbf{f}) .
\end{aligned}
$$

For any choice of a qubit-model Qub, the syntactical tree of $\alpha$ determines a corresponding sequence of quregisters. Consider a sentence $\alpha$ with $n$ atomic occurrences $\left(\mathbf{q}_{1}, \ldots, \mathbf{q}_{n}\right)$. Then, Qub $(\alpha) \in \otimes^{n} \mathbb{C}^{2}$ (where $\otimes^{n} \mathbb{C}^{2}$ represents the semantic space $\mathcal{H}^{\alpha}$ of $\alpha$ ). We can associate a quregister $\mid \psi_{i}$ to each $\operatorname{Level}_{i}(\alpha)$ of $S \operatorname{STe} e^{\alpha}$ in the following way. Suppose that:

$$
\operatorname{Level}_{i}(\alpha)=\left(\beta_{1}, \ldots, \beta_{r}\right) .
$$

Then:

$$
\left|\psi_{i}\right\rangle=\operatorname{Qub}\left(\beta_{1}\right) \otimes \cdots \otimes \operatorname{Qub}\left(\beta_{r}\right) .
$$

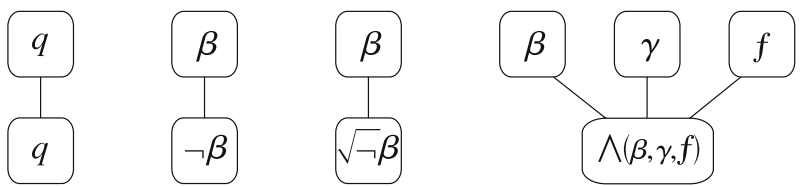

Figure 1. Branching rules for the construction of syntactical trees. 
Instead of $\operatorname{Qub}\left(\beta_{1}\right) \otimes \cdots \otimes \operatorname{Qub}\left(\beta_{\mathrm{r}}\right)$, we will briefly write $\operatorname{Qub}\left(\right.$ Level $\left._{i}(\alpha)\right)$. Notice that all $\operatorname{Qub}\left(\operatorname{Level}_{i}(\alpha)\right)$ belong to the space $\mathcal{H}^{\alpha}$.

The notion of qubit tree of a sentence $\alpha\left(\right.$ QubTree $\left.^{\alpha}\right)$ can be now defined as a particular sequence of unitary operators that is uniquely determined by the syntactical tree of $\alpha$. Let Level $_{i}^{j}(\alpha)$ represent the $j$ th element of $\operatorname{Level}_{i}(\alpha)$. Each node $\operatorname{Level}_{i}^{j}(\alpha)$ (where $1 \leq i<\operatorname{Height}(\alpha)$ ) can be naturally associated to a unitary operator $O p_{i}^{j}$, according to the following operator-rule:

$$
O p_{i}^{j}:=\left\{\begin{array}{cl}
I^{(1)} & \text { if } \text { Level }_{i}{ }^{j}(\alpha) \text { is an atomic sentence; } \\
\operatorname{Not}^{(r)} & \text { if } \text { Level }_{i}{ }^{j}(\alpha)=\neg \beta \text { and Qub }(\beta) \in \otimes^{\mathrm{r}} \mathbb{C}^{2} ; \\
\sqrt{\operatorname{Not}}^{(r)} & \text { if } \text { Level }_{i}{ }^{j}(\alpha)=\sqrt{\neg} \beta \text { and } \operatorname{Qub}(\beta) \in \otimes^{\mathrm{r}} \mathbb{C}^{2} ; \\
\mathrm{T}^{(r, s, 1)} & \text { if } \text { Level }_{i}{ }^{j}(\alpha)=\bigwedge(\beta, \gamma, \mathbf{f}), \operatorname{Qub}(\beta) \in \otimes^{r} \mathbb{C}^{2} \text { and } \operatorname{Qub}(\gamma) \in \otimes^{s} \mathbb{C}^{2},
\end{array}\right.
$$

where $I^{(1)}$ is the identity operator of $\mathbb{C}^{2}$.

On this basis, one can associate an operator $U_{i}$ to each $\operatorname{Level}_{i}(\alpha)$ (such that $1 \leq i<\operatorname{Height}(\alpha)$ ):

$$
U_{i}:=\bigotimes_{j=1}^{\mid \text {Level }_{i}(\alpha) \mid} O p_{i}^{j}
$$

where $\left|\operatorname{Level}_{i}(\alpha)\right|$ is the length of the sequence $\operatorname{Level}_{i}(\alpha)$.

Being the tensor product of unitary operators, every $U_{i}$ turns out to be a unitary operator. One can easily show that all $U_{i}$ are defined on the same space, $\mathcal{H}^{\alpha}$.

Definition 5.1 (The qubit tree of $\alpha$ ). The qubit tree of $\alpha$ (denoted by $Q_{T r e e^{\alpha}}$ ) is the operator-sequence

$$
\left(U_{1}, \ldots, U_{\text {Height }(\alpha)-1}\right)
$$

that is uniquely determined by the syntactical tree of $\alpha$.

As an example, consider again the sentence: $\alpha=\bigwedge(\mathbf{q}, \neg \mathbf{q}, \mathbf{f})$.

In order to construct the qubit tree of $\alpha$, let us first determine the operators $O p_{i}^{j}$ corresponding to each node of Stree $e^{\alpha}$. We will obtain:

- $O p_{1}^{1}=\mathrm{T}^{(1,1,1)}$, because $\bigwedge(\mathbf{q}, \neg \mathbf{q}, \mathbf{f})$ is connected with $(\mathbf{q}, \neg \mathbf{q}, \mathbf{f})$ (at Level $_{2}(\alpha)$;

- $O p_{2}^{1}=I^{(1)}$, because $\mathbf{q}$ is connected with $\mathbf{q}$ (at Level $_{3}(\alpha)$ );

- $O p_{2}^{2}=\operatorname{Not}^{(1)}$, because $\neg \mathbf{q}$ is connected with $\mathbf{q}$ (at Level $(\alpha)$ );

- $O p_{2}^{3}=I^{(1)}$, because $\mathbf{f}$ is connected with $\mathbf{f}\left(\right.$ at $\left.\operatorname{Level}_{3}(\alpha)\right)$. 
The qubit tree of $\alpha$ is represented by the operator-sequence $\left(U_{1}, U_{2}\right)$, where:

$$
\begin{aligned}
& U_{1}=O p_{1}^{1}=\mathrm{T}^{(1,1,1)} \\
& U_{2}=O p_{2}^{1} \otimes O p_{2}^{2} \otimes O p_{2}^{3}=I^{(1)} \otimes \operatorname{Not}^{(1)} \otimes \mathrm{I}^{(1)} .
\end{aligned}
$$

Apparently, QTree ${ }^{\alpha}$ is independent of the choice of Qub.

Theorem 5.1 Let $\alpha$ be a sentence whose qubit tree is the operator-sequence $\left(U_{1}, \ldots, U_{\text {Height( }(\alpha)-1}\right)$, and let Qub be a qubit-model. Then,

$$
U_{i}\left(\operatorname{Qub}\left(\operatorname{Level}_{i+1}(\alpha)\right)=\operatorname{Qub}\left(\operatorname{Level}_{i}(\alpha)\right)\right.
$$

(for any $i$ such that $1 \leq i<\operatorname{Height}(\alpha)$ ).

The qubit tree of $\alpha$ can be naturally regarded as a quantum circuit that computes the output $\operatorname{Qub}(\alpha)$, given the input $\operatorname{Qub}\left(\mathbf{q}_{1}\right), \ldots, \operatorname{Qub}\left(\mathbf{q}_{n}\right)$ (where $\mathbf{q}_{1}, \ldots, \mathbf{q}_{n}$ are the atomic occurrences of $\alpha$ ). In this framework, each $U_{i}$ is the unitary operator that describes the computation performed by the $i$ th layer of the circuit.

Let us now turn to the notion of qumix tree. Consider a qumixmodel Qum and let $\alpha$ be a sentence such that $\operatorname{At}(\alpha)=n$ and $\operatorname{Height}(\alpha)=k$. Let $U_{1}, \ldots, U_{k-1}$ be the qubit tree of $\alpha$. Suppose that Level $_{i}(\alpha)=\beta_{1}, \ldots, \beta_{r}$. Like in the case of qubit-models, we will briefly writeQum $\left(\operatorname{Level}_{i}(\alpha)\right)$ for $\operatorname{Qum}\left(\beta_{1}\right) \otimes \cdots \otimes \operatorname{Qum}\left(\beta_{\mathrm{r}}\right)$. We can now define the following sequence of functions on the set $\mathfrak{D}\left(\mathcal{H}^{\alpha}\right)$ :

$$
\begin{gathered}
{ }^{\mathcal{D}} U_{1}(\rho)=U_{1} \rho U_{1}^{*} \\
\cdots \\
{ }^{\mathcal{D}} U_{k-1}(\rho)=U_{k-1} \rho U_{k-1}^{*} .
\end{gathered}
$$

Lemma 5.1 For any $\rho \in \mathfrak{D}\left(\mathcal{H}^{\alpha}\right),{ }^{\mathcal{D}} \mathcal{U}_{\infty}(\rho)$ is a density operator of $\mathfrak{D}\left(\mathcal{H}^{\alpha}\right)$.

Lemma 5.2 ${ }^{\mathcal{D}} U_{i}\left(\operatorname{Qum}\left(\operatorname{Level}_{i+1}(\alpha)\right)\right)=\operatorname{Qum}_{\left(\operatorname{Level}_{i}(\alpha)\right) .}$

The sequence $\left({ }^{\mathcal{D}} U_{1}, \ldots,{ }^{\mathcal{D}} U_{k-1}\right)$ (obtained from the qubit tree $\left.\left(U_{1}, \ldots, U_{k-1}\right)\right)$ is called the qumix tree of $\alpha$ (and indicated by Qum$\left.\operatorname{Tree}^{\alpha}\right)$. 


\section{Holistic semantics}

In the compositional semantics, the meaning of a molecular sentence is determined by the meanings of its parts. As a consequence, in this framework, the meaning of a molecular $\alpha$ cannot be a pure state, when some atomic parts of $\alpha$ are proper mixtures. How to generalize the quantum computational semantics in order to represent some typical quantum holistic situations, including the possibility of entangled meanings? ${ }^{1}$

As we have seen, any qumix-model assigns to the top-level of the syntactical tree of a sentence $\alpha$ a factorized meaning, Qum $\left(\mathbf{q}_{1}\right) \otimes \cdots \otimes$ $\operatorname{Qum}\left(\mathbf{q}_{n}\right)$ (where $\mathbf{q}_{1}, \ldots, \mathbf{q}_{n}$ are the atomic sentences occurring in $\alpha$ ). The holistic semantics ${ }^{2}$ is based on the following "more liberal" assumption: the meaning of the top-level of the syntactical tree of a sentence $\alpha$ might be any qumix (not necessarily factorized) "living" in the semantic space of $\alpha$. As a consequence, also the meanings of all other levels of $S T r e e^{\alpha}$ are not necessarily factorized.

Suppose that:

$$
\operatorname{Level}_{i}(\alpha)=\left(\beta_{1}, \ldots, \beta_{r}\right) .
$$

The space $\mathcal{H}^{\alpha}$ can be naturally regarded as the Hilbert space of a compound physical system consisting of $r$ parts (mathematically represented by the spaces $\left.\mathcal{H}^{\beta_{1}}, \ldots, \mathcal{H}^{\beta_{r}}\right)$, where each part may be compound. On this basis, for any qumix $\rho_{i}$ (associated to $\operatorname{Level}_{i}(\alpha)$ ) and for any node Level $_{i}^{j}(\alpha)$, we can consider the reduced state red ${ }^{j}\left(\rho_{i}\right)$ with respect to the $j$ th subsystem of the system described by $\rho_{i}$. From an intuitive point of view, $\operatorname{red}^{j}\left(\rho_{i}\right)$ describes the $j$ th subsystem on the basis of the global information $\rho_{i}$. We recall that $\operatorname{red}^{j}\left(\rho_{i}\right)$ is the unique density operator that satisfies the following condition: for any self-adjoint operator $A^{j}$ of $\mathcal{H}^{\beta_{j}}$,

$$
\operatorname{tr}\left(\operatorname{red}^{j}\left(\rho_{i}\right) A^{j}\right)=\operatorname{tr}\left(\rho_{i}\left(I^{1} \otimes \cdots \otimes I^{j-1} \otimes A^{j} \otimes I^{j+1} \otimes \cdots \otimes I^{r}\right)\right),
$$

where $I^{h}$ is the identity operator of $\mathcal{H}^{\beta_{h}}$.

As a consequence, we obtain that $\rho_{i}$ and $\operatorname{red}^{j}\left(\rho_{i}\right)$ are statistically equivalent with respect to the $j$ th subsystem of the compound system described by $\rho_{i}$.

Since $\operatorname{Level}_{i}(\alpha)=\left(\beta_{1}, \ldots, \beta_{r}\right)$, the qumix $\operatorname{red}^{j}\left(\rho_{i}\right)$ (which is a density operator of the space $\mathcal{H}^{\beta_{j}}$ ) can be regarded as a possible meaning of the sentence $\beta_{j}$. 
We can now introduce the basic definitions of the holistic semantics.

Definition 6.1 (Atomic holistic model). An atomic holistic model is a map $\mathrm{Hol}^{A t}$ that associates a qumix to any sentence $\alpha$ of $\mathcal{L}$, satisfying the following conditions:

(1) $\operatorname{Hol}^{A t}(\alpha) \in \mathfrak{D}\left(\mathcal{H}^{\alpha}\right)$;

(2) Let $\operatorname{At}(\alpha)=n$ and Level Height $(\alpha)=\mathbf{q}_{1}, \ldots, \mathbf{q}_{n}$. Then,

(2.1) $\mathbf{q}_{j}=\mathbf{f} \Rightarrow \operatorname{red}^{j}\left(\mathrm{Hol}^{A t}(\alpha)\right)=P_{0}$;

(2.2) if $\mathbf{q}_{j}$ and $\mathbf{q}_{h}$ are two occurrences in $\alpha$ of the same atomic sentence, then $\operatorname{red}^{j}\left(\mathrm{Hol}^{A t}(\alpha)\right)=\operatorname{red}^{h}\left(\operatorname{Hol}^{A t}(\alpha)\right)$.

Apparently, $\mathrm{Hol}^{A t}(\alpha)$ represents a global interpretation of the atomic sentences occurring in $\alpha$. At the same time, $\operatorname{red}^{j}\left(\mathrm{Hol}^{A t}(\alpha)\right)$, the reduced state of the compound system (described by $\mathrm{Hol}^{A t}(\alpha)$ ) with respect to the $j$ th subsystem, represents a contextual meaning of $\mathbf{q}_{j}$ with respect to the global meaning $\mathrm{Hol}^{A t}(\alpha)$. Conditions (2.1) and (2.2) guarantee that $\mathrm{Hol}^{A t}(\alpha)$ is well behaved. For, the contextual meaning of $\mathbf{f}$ is always the Falsity, while two different occurrences (in $\alpha$ ) of the same atomic sentence have the same contextual meaning.

The map $\mathrm{Hol}^{A t}$ (which assigns a meaning to the top-level of the syntactical tree of any sentence $\alpha$ ) can be naturally extended to a map $\mathrm{Hol}^{\text {Tree }}$ that assigns a meaning to each level of the syntactical tree of any $\alpha$, following the prescriptions of the qumix tree of $\alpha$.

Consider a sentence $\alpha$ such that:

$$
\operatorname{QumTree}^{\alpha}=\left({ }^{\mathcal{D}} U_{1}, \ldots,{ }^{\mathcal{D}} U_{\text {Height }(\alpha)-1}\right) \text {. }
$$

The map Hol ${ }^{\text {Tree }}$ is defined as follows:

$$
\begin{aligned}
& \operatorname{Hol}^{\text {Tree }}\left(\text { Level }_{\text {Height }(\alpha)}\right)=\operatorname{Hol}^{\text {At }}(\alpha) \\
& \operatorname{Hol}^{\text {Tree }}\left(\text { Level }_{i}(\alpha)\right)={ }^{\mathcal{D}} U_{i}\left(\operatorname{Hol}^{\text {Tree }}\left(\text { Level }_{i+1}(\alpha)\right)\right.
\end{aligned}
$$

where $\operatorname{Height}(\alpha)>(i \geq 1)$.

On this basis, one can naturally define the notion of holistic model of $\mathcal{L}$. 
Definition 6.2 (Holistic model). A holistic model of $\mathcal{L}$ is a map Hol that assigns to any sentence $\alpha$ a qumix of the space $\mathcal{H}^{\alpha}$, according to the following condition:

$$
\operatorname{Hol}(\alpha)=\operatorname{Hol}^{\text {Tree }}\left(\text { Level }_{1}(\alpha)\right)
$$

Given a sentence $\gamma$, Hol determines the contextual meaning, with respect to the context $\mathrm{Hol}(\gamma)$, of any occurrence of a subformula $\beta$ in $\gamma$.

Definition 6.3 (Contextual meaning of a node). Let $\beta$ be a subformula of $\gamma$ occurring at the $j$ th position of the $i$ th level of the syntactical tree of $\gamma$. We indicate by $\beta\left[{ }_{i}^{i}\right]$ the node of STree ${ }^{\gamma}$ corresponding to such occurrence. The contextual meaning of $\beta\left[{ }_{j}^{i}\right]$ with respect to the context $\mathrm{Hol}(\gamma)$ is defined as follows:

$$
\left.\operatorname{Hol}^{\gamma}\left(\beta_{j}^{i}\right]\right)=\operatorname{red}^{j}\left(\operatorname{Hol}^{\text {Tree }}\left(\operatorname{Level}_{i}(\gamma)\right)\right)
$$

Hence, we have:

$$
\operatorname{Hol}^{\gamma}(\gamma)=\operatorname{Hol}^{\text {Tree }}\left(\text { Level }_{1}(\gamma)\right)=\operatorname{Hol}(\gamma)
$$

\section{Lemma 6.1}

1. Let $\beta\left[\begin{array}{l}i \\ j\end{array}\right]$ be a node of $S T r e e^{\gamma}$ and let $\neg \beta\left[\begin{array}{c}i-1 \\ h\end{array}\right]$ be the node of STree obtained by applying the negation $\neg$ to the occurrence of $\beta$ at the node $\beta\left[\begin{array}{l}i \\ j\end{array}\right]$. Then,

$$
\operatorname{Hol}^{\gamma}\left(\neg \beta\left[\begin{array}{l}
i-1 \\
h
\end{array}\right]\right)=\operatorname{NOT}\left(\operatorname{Hol}^{\gamma}\left(\beta\left[\begin{array}{l}
i \\
j
\end{array}\right]\right)\right) .
$$

2. Let $\beta\left[\begin{array}{l}i \\ j\end{array}\right]$ be a node of $S T r e e^{\gamma}$ and let $\sqrt{\neg} \beta\left[\begin{array}{c}i-1 \\ h\end{array}\right]$ be the node of STree $e^{\gamma}$ obtained by applying the square root of the negation $\sqrt{\neg}$ to the occurrence of $\beta$ at the node $\beta\left[{ }_{j}^{i}\right]$. Then,

$$
\operatorname{Hol}^{\gamma}\left(\sqrt{\neg} \beta\left[\begin{array}{c}
i-1 \\
h
\end{array}\right]\right)=\sqrt{\mathrm{NOT}}\left(\operatorname{Hol}^{\gamma}\left(\beta\left[\begin{array}{l}
i \\
j
\end{array}\right]\right)\right) .
$$

3. Let $\left.\beta\left[{ }_{j}^{i}\right], \quad \delta\left[{ }_{j+1}^{i}\right], \quad \mathbf{f}^{i}{ }_{j+2}^{i}\right]$ be three nodes of STree ${ }^{\gamma}$ and let $\bigwedge(\beta, \delta, \mathbf{f})\left[\begin{array}{c}i-1 \\ h\end{array}\right]$ be the node of STree ${ }^{\gamma}$ obtained by applying the 
conjunction $\Lambda$ to the occurrences of $\beta, \delta, \mathbf{f}$ at the nodes $\beta\left[{ }_{j}^{i}\right]$, $\delta\left[\begin{array}{c}i \\ j+1\end{array}\right], \mathbf{f}\left[\begin{array}{c}i \\ j+2\end{array}\right]$. Then,

$$
\begin{aligned}
\operatorname{Hol}^{\gamma}\left(\bigwedge(\beta, \delta, \mathbf{f})\left[\begin{array}{c}
i-1 \\
h
\end{array}\right]\right)= & \mathbb{T}\left(\operatorname{Hol}^{\gamma}\left(\beta\left[\begin{array}{l}
i \\
j
\end{array}\right]\right), \operatorname{Hol}^{\gamma}\left(\delta\left[\begin{array}{c}
i \\
j+1
\end{array}\right]\right),\right. \\
& \left.\operatorname{Hol}^{\gamma}\left(\mathbf{f}\left[\begin{array}{c}
i \\
j+2
\end{array}\right]\right)\right) .
\end{aligned}
$$

Lemma 6.2 If $\beta\left[\begin{array}{l}i \\ j\end{array}\right]$ and $\beta\left[\begin{array}{l}h \\ k\end{array}\right]$ are two nodes of the syntactical tree of $\gamma$, representing two occurrences of the same subformula $\beta$, then:

$$
\operatorname{Hol}^{\gamma}\left(\beta\left[\begin{array}{l}
i \\
j
\end{array}\right]\right)=\operatorname{Hol}^{\gamma}\left(\beta\left[\begin{array}{l}
h \\
k
\end{array}\right]\right)
$$

In other words, two different occurrences of one and the same subformula in a sentence $\gamma$ receive the same contextual meaning with respect to the context $\mathrm{Hol}(\gamma)$.

On this basis, one can define the contextual meaning of a subformula $\beta$ of $\gamma$, with respect to the context $\operatorname{Hol}(\gamma)$ :

$$
\operatorname{Hol}^{\gamma}(\beta):=\operatorname{Hol}^{\gamma}\left(\beta\left[\begin{array}{l}
i \\
j
\end{array}\right]\right) \text {, }
$$

where $\beta\left[{ }_{j}^{i}\right]$ is any occurrence of $\beta$ at a node of $S T r e e^{\gamma}$.

Suppose now that $\beta$ is a subformula of two different formulas $\gamma$ and $\delta$. Generally, we have:

$$
\operatorname{Hol}^{\gamma}(\beta) \neq \operatorname{Hol}^{\delta}(\beta) \text {. }
$$

In other words, sentences may receive different contextual meanings in different contexts!

Apparently, $\mathrm{Hol}^{\gamma}$ is a partial function that only assigns meanings to the subformulas of $\gamma$. Given a sentence $\gamma$, we will call the partial functionHol ${ }^{\gamma}$ a contextual holistic model of the language.

Lemma 6.3 Any compositional model Qum uniquely determines a holistic model Hol such that:

1. $\operatorname{Qum}(\alpha)=\operatorname{Hol}(\alpha)$, for any sentence $\alpha$;

2. $\operatorname{Qum}(\alpha)=\operatorname{Hol}^{\gamma}(\alpha)$, for any $\gamma$ such that $\alpha$ is a subformula of $\gamma$. 
Proof. Given Qum, define $\mathrm{Hol}^{A t}$ as follows. For any $\alpha$ s.t. Level ${ }_{\text {Height }(\alpha)}$ $=\mathbf{q}_{1}, \ldots, \mathbf{q}_{n}$,

$$
\operatorname{Hol}^{A t}(\alpha)=\operatorname{Qum}\left(\mathbf{q}_{1}\right) \otimes \cdots \otimes \operatorname{Qum}\left(\mathbf{q}_{n}\right)
$$

We can now define the notion of logical consequence in the holistic semantics.

Definition 6.4 (Consequence in a given contextual model $\mathrm{Hol}^{\gamma}$ ). A sentence $\beta$ is a consequence of a sentence $\alpha$ in a given contextual model $\operatorname{Hol}^{\gamma}\left(\alpha \models_{\mathrm{Hol}^{\gamma}} \beta\right)$ iff

1. $\alpha$ and $\beta$ are subformulas of $\gamma$;

2. $\operatorname{Hol}^{\gamma}(\alpha) \preceq \operatorname{Hol}^{\gamma}(\beta)$.

Definition 6.5 (Logical consequence (in the holistic semantics)). A sentence $\beta$ is a consequence of a sentence $\alpha$ (in the holistic semantics) $(\alpha \not \models \beta)$ iff for any sentence $\gamma$ such that $\alpha$ and $\beta$ are subformulas of $\gamma$ and for any Hol,

$$
\alpha \models_{\mathrm{Hol}^{\gamma}} \beta \text {. }
$$

We call HQCL the logic that is semantically characterized by the logical consequence relation we have just defined. Hence, $\alpha \nvdash_{\text {HQCL }} \beta$ iff for any sentence $\gamma$ such that $\alpha$ and $\beta$ are subformulas of $\gamma$ and for any $\mathrm{Hol}$,

$$
\alpha \models_{\mathrm{Hol}^{\gamma}} \beta .
$$

Theorem 6.1 HQCL and QCL are the same logic.

\section{Proof.}

1. $\alpha \models_{\text {HQCL }} \beta \Rightarrow \alpha \models_{\mathbf{Q C L}} \beta$. Suppose $\alpha \models_{\text {HQCL }} \beta$ and $\alpha \nvdash_{\mathbf{Q C L}} \beta$. Then, there exists a compositional model Qum, such that: Qum $(\alpha) \npreceq$ Qum $(\beta)$. By Lemma 6.3, there exists a holistic model Hol such that: 


$$
\operatorname{Hol}^{\alpha \wedge \beta}(\alpha)=\operatorname{Qum}(\alpha), \operatorname{Hol}^{\alpha \wedge \beta}(\beta)=\operatorname{Qum}(\beta) .
$$

Hence, $\operatorname{Hol}^{\alpha \wedge \beta}(\alpha) \npreceq \operatorname{Hol}^{\alpha \wedge \beta}(\beta)$, against the hypothesis!

2. $\alpha \models_{\mathbf{Q C L}} \beta \Rightarrow \alpha \models_{\mathbf{H Q C L}} \beta$. Suppose $\alpha \models_{\mathbf{Q C L}} \beta$ and $\alpha \nvdash_{\mathbf{H Q C L}} \beta$. Then, there exist a holistic model $\mathrm{Hol}$ and a sentence $\gamma$ that contains $\alpha$ and $\beta$ as subformulas, such that:

$$
\operatorname{Hol}^{\gamma}(\alpha) \npreceq \operatorname{Hol}^{\gamma}(\beta) \text {. }
$$

Consider STree ${ }^{\gamma}$ and suppose that:

$$
\text { Level }_{\text {Height }(\gamma)}=\mathbf{q}_{1}, \ldots, \mathbf{q}_{n} .
$$

Define the following compositional model Qum:

$$
\operatorname{Qum}\left(\mathbf{q}_{j}\right)=\left\{\begin{array}{cc}
\operatorname{Hol}^{\gamma}\left(\mathbf{q}_{j}\right), & \text { if } \mathbf{q}_{j} \in\left\{\mathbf{q}_{1}, \ldots, \mathbf{q}_{n}\right\} \\
P_{0}, & \text { otherwise. }
\end{array}\right.
$$

Lemma 6.4 If $\delta$ is a subformula of $\gamma$ then

$$
\operatorname{Hol}^{\gamma}(\delta) \preceq \operatorname{Qum}(\delta) ; \operatorname{Qum}(\delta) \preceq \operatorname{Hol}^{\gamma}(\delta) \text {. }
$$

As a consequence of Lemma 6.4, we obtain:

$$
\operatorname{Qum}(\alpha) \npreceq \operatorname{Qum}(\beta),
$$

because $\alpha$ and $\beta$ are subformulas of $\gamma$. Against the hypothesis!

\section{Notes}

1 The basic features of an entangled state $|\Psi\rangle$ can be sketched as follows: (1) $\mid \Psi>$ is a maximal information (a pure state) that describes a compound physical system $S$; (2) the pieces of information determined by $\mid \Psi>$ about the parts of $S$ are, generally, non-maximal (proper mixtures). Hence, the information about the whole is more precise than the information about the parts.

2 In Dalla Chiara et al. (2005) we have presented a weaker version of holistic semantics.

\section{References}

Birkhoff $G$ and Neumann Jvon (1936) The logic of quantum mechanics. Annals of Mathematics 37: 823-843 
Dalla Chiara ML, Giuntini R and Leporini R (2003) Quantum computational logics. A survey. In: Hendricks V and Malinowski J (eds) Trends in Logic. 50 Years of StudiaLogica, pp. 229-271. Kluwer

Dalla Chiara ML, Giuntini R and Leporini R (2004a) Quantum computational logics and Fock space semantics. International Journal of Quantum Information 2: 1-8

Dalla Chiara ML, Giuntini R and Leporini R (2005) Logics from quantum computation. International Journal of Quantum Information 3: 293-337

Dalla Chiara ML and Giuntini R (2002) Quantum logics. In: Gabbay G and Guenthner F (eds), Handbook of Philosophical Logic, Vol. VI Kluwer, Dordrecht pp. 129-228.

Dalla Chiara ML, Giuntini R and Greechie R (2004b) Reasoning in Quantum Theory. Kluwer, Dordrecht

Deutsch D, Ekert A and Lupacchini R (2000) Machines, logic and quantum physics. Bulletin of Symbolic Logic 3: 265-283

Gudder S (2003) Quantum computational logic. International Journal of Theoretical Physics 42: 39-47 